\title{
Tarsal Coalitions in Adults: A Case Series
}

\author{
Rajiv Shah ${ }^{1}$, Nikku Mathew Geevarughese ${ }^{2}$, Shivam R Shah ${ }^{3}$
}

\begin{abstract}
Tarsal coalition is an uncommon finding in children and adolescent age groups. Its occurrence in adulthood is even rarer. Though the majority of the coalitions are asymptomatic, pain, and fatigue in $25 \%$ of coalitions become indicators for aggressive treatment. Surgical procedures are undertaken only at the failure of conservative management. Triple arthrodesis has been advocated earlier but causes substantial stresses at the ankle joint. Hence, a limited arthrodesis aiming at the involved joint spares loading of the ankle and calcaneocuboid joints. More so, hindfoot valgus is a common component noted in tarsal coalitions and needs to be addressed along with isolated arthrodesis to achieve overall favorable outcomes. We present a series of tarsal coalitions in adults who presented with different symptoms, managed with corrective arthrodesis, and simultaneous treatment of associated pes planus deformity.
\end{abstract}

Keywords: Calcaneonavicular coalition, Double arthrodesis, Gastrocnemius recession, Talocalcaneal coalition, Talonavicular arthrodesis, Talonavicular coalition, Tarsal coalition, Triple coalition.

Journal of Foot and Ankle Surgery (Asia Pacific) (2021): 10.5005/jp-journals-10040-1138

\section{Aım}

This study aimed to understand the radiological and functional outcome of the tarsal coalitions in adults treated with selective arthrodesis and deformity correction procedures for accompanying pes planovalgus deformity.

\section{BACKGROUND}

Tarsal coalition is an unusual bony or cartilaginous union between bones of the hindfoot. Talocalcaneal and calcaneonavicular coalitions are more common and cause significant hindfoot pain. ${ }^{1,2}$ Usually developing in the adolescent age group, coalitions ossify by adulthood. Pain is the usual presenting symptom and, secondly, a flatfoot. Symptomatic treatment is attained with antiinflammatories and orthotics or footwear modifications. Operative treatment is indicated after failure of conservative treatment, for which options include resection, resection and interposition, or arthrodesis. ${ }^{3}$ In situations where multiple coalitions are present with arthritic changes set in, arthrodesis yield better functional outcomes. Here, we present a case series of five different instances of symptomatic tarsal coalitions in the adult age who presented with varied symptoms approached by case-based surgical procedures.

\section{Case Description}

The initial examination included assessing deformities of the forefoot and midfoot, medial arch, heel valgus or varus deformity, and ascertaining whether the deformities are fixed or flexible. Due importance was given for points of tenderness. Peroneal and tibialis posterior tendons were screened for any signs of inflammation. Gastrosoleus tightness was assessed. Active and passive movements at the subtalar and midtarsal joints were assessed and compared with the opposite side.

Radiographic evaluation begins with weight-bearing anteroposterior and $45^{\circ}$ medial oblique view of the foot and anteroposterior and lateral views of the ankle. Imaging of both the feet is taken for comparison to detect an asymptomatic coalition on the opposite side. A detailed description of clinical and radiological findings in all our cases is mentioned and summarized
${ }^{1}$ Sunshine Global Hospitals, Vadodara, Gujarat, India

${ }^{2}$ Department of Orthopaedics, All India Institute of Medical Sciences, Bhopal, Madhya Pradesh, India

${ }^{3}$ Department of Orthopaedics, Baroda Medical College, Vadodara, Gujarat, India

Corresponding Author: Nikku Mathew Geevarughese, Department of Orthopaedics, All India Institute of Medical Sciences, Bhopal, Madhya Pradesh, India, Phone: +91 9632599776, e-mail: nikku.mathew@gmail. com

How to cite this article: Shah R, Geevarughese NM, Shah SR. Tarsal Coalitions in Adults: A Case Series. J Foot Ankle Surg (Asia Pacific) 2021;8(2):42-47.

Source of support: Nil

Conflict of interest: None

in Table 1. American Orthopaedic Foot and Ankle Society (AOFAS) ankle hindfoot score was recorded for all patients, both preoperatively and at the final follow-up. Informed consent was obtained from every patient. Permission from the hospital review board was also obtained. Case-specific details follow.

\section{Case 1}

$H D$, a 24-year-old woman, presented with severe stiffness along with ankle pain and fatigue for 3 years. On evaluation, she had a planovalgus foot with absent movements at subtalar and transverse tarsal joints (Fig. 1). X-rays confirmed planus foot and heel in valgus. X-rays also showed the presence of triple coalition (talocalcaneal, calcaneocuboid, and talonavicular) without arthritic changes in surrounding joints (Fig. 2). As the patient had undergone conservative treatment for 3 years in the form of orthotics and footwear modifications and analgesics, we decided to undertake surgical management. She underwent corrective open wedge arthrodesis at the talonavicular coalition, at the apex of flat foot deformity. An ipsilateral iliac crest bone graft was used. She also underwent gastrocnemius recession and medial calcaneal slide osteotomy fixed with two cannulated cancellous screws. Screws were pushed up to the neck and body of the talus for better 
Table 1: Complete case details of all five cases

\begin{tabular}{|c|c|c|c|c|c|c|c|}
\hline \multirow[b]{2}{*}{ No." } & \multirow[b]{2}{*}{ Age $e^{*}$ and sex } & \multirow[b]{2}{*}{ Side } & \multirow[b]{2}{*}{ Diagnosis } & \multirow[b]{2}{*}{ Procedures } & \multirow{2}{*}{$\begin{array}{l}\text { Follow-up (in } \\
\text { months) }\end{array}$} & \multicolumn{2}{|c|}{ AOFAS score } \\
\hline & & & & & & Pre-op & Post-op \\
\hline 1 & $F, 24$ & $\mathrm{R}$ & $\begin{array}{l}\mathrm{TC}+\mathrm{CC}+\mathrm{TN} \text { coalition }+ \text { heel } \\
\text { valgus }\end{array}$ & $\begin{array}{l}\text { TN fusion + MCSO + gastrocnemius } \\
\text { recession }\end{array}$ & 18 & 46 & 72 \\
\hline 2 & $M, 18$ & L & $\begin{array}{l}\text { TC }+ \text { CN coalition }+ \text { Non- } \\
\text { healing ulcer }+ \text { heel valgus }\end{array}$ & $\begin{array}{l}\text { TN fusion + MCSO + gastrocnemius } \\
\text { recession }\end{array}$ & 24 & 58 & 76 \\
\hline 3 & $M, 26$ & L & TC coalition + heel valgus & $\begin{array}{l}\text { TN fusion + MCSO + gastrocnemius } \\
\text { recession }\end{array}$ & 20 & 64 & 82 \\
\hline 4 & $\mathrm{~F}, 40$ & L & $\begin{array}{l}\text { TC coalition + talonavicular } \\
\text { osteophyte }\end{array}$ & $\begin{array}{l}\text { TN joint cheilectomy + gastrocnemius } \\
\text { recession }\end{array}$ & 36 & 60 & 78 \\
\hline 5 & $M, 32$ & L & $\begin{array}{l}\text { TC coalition }+ \text { heel valgus }+ \\
\text { forefoot abduction }\end{array}$ & $\begin{array}{l}\text { TN fusion + Evans osteotomy + MCSO } \\
+ \text { gastrocnemius recession }\end{array}$ & 24 & 58 & 76 \\
\hline
\end{tabular}

\#No. means a number allocated to the case

*Age is described in years

F, female; M, male; R, right; L, left; TC, talocalcaneal; CC, calcaneocuboid; TN, talonavicular; CN, calcaneonavicular; MCSO, medial calcaneal slide osteotomy
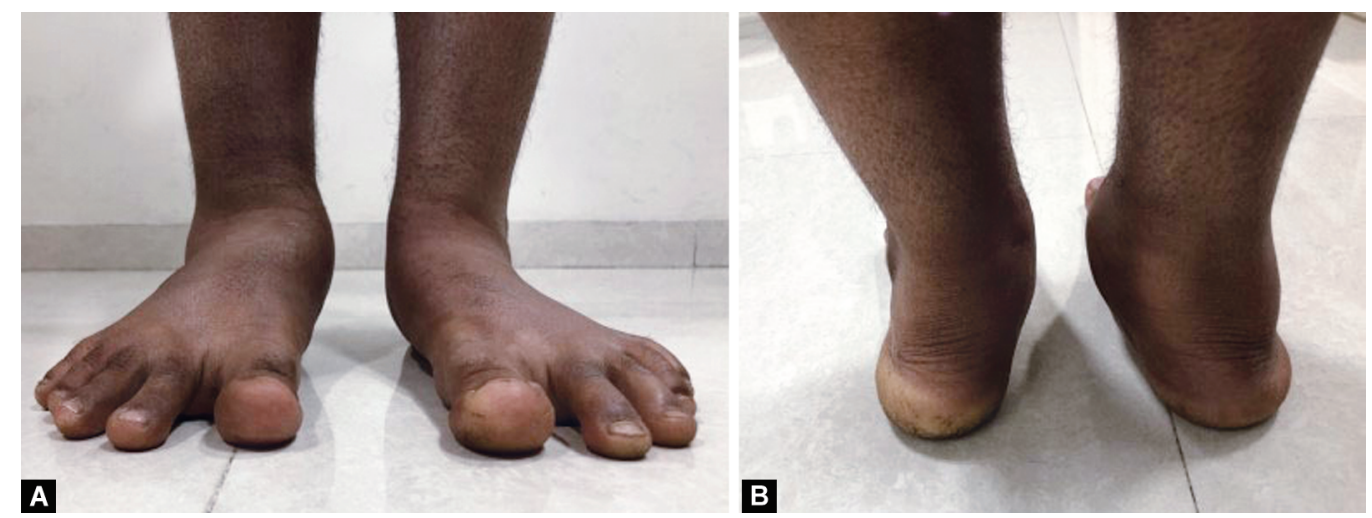

Figs 1 A and B: Case 1, HD, a 24-year-old woman with right planovalgus deformity and rigid flatfoot

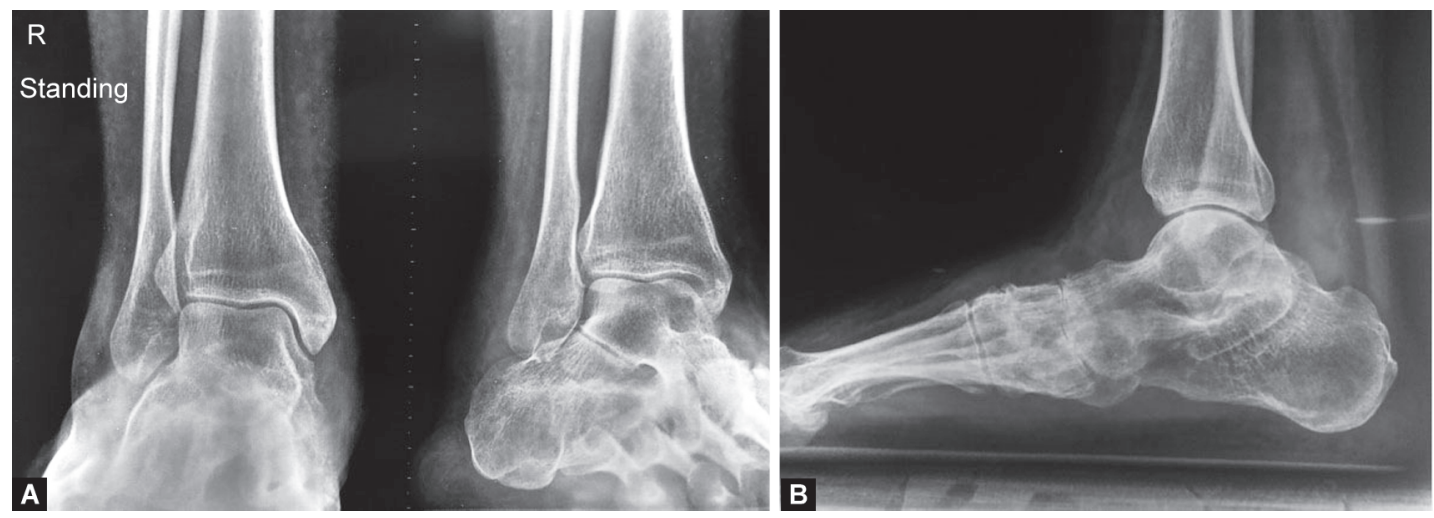

Figs $2 \mathrm{~A}$ and B: Case 1, preoperative radiograph demonstrating congenital coalition of talocalcaneal, calcaneocuboid, and talonavicular joints

stability (Fig. 3). Postoperatively, her mechanics were found to be corrected aptly, and at 18 months follow-up, she showed good results with improved AOFAS score from 46 preoperatively to 72 postoperatively.

\section{Case 2}

AG, an 18-year-old man, presented with a non-healing ulcer over the medial aspect of the left ankle for the past 2 years, failing multiple treatments. On evaluation, the patient had severe planovalgus foot with stretched medial skin over the ankle (Fig. 4), preventing healing of the ulcer every time he walked. X-rays showed talocalcaneal and calcaneonavicular coalitions with arthritic changes and dorsal osteophytes at the talonavicular joint with a drop of talar head on the weight-bearing views (Fig. 5).

The patient underwent a gastrocnemius recession, medial calcaneal slide osteotomy, and talonavicular arthrodesis. Arthrodesis and osteotomy, both were fixed with $5 \mathrm{~mm}$ cannulated cancellous screws. We harvested bone grafts from the proximal tibia for arthrodesis. Osteotomy screws were pushed up to the neck and body of the talus for better stability (Fig. 6). His non-healing ulcer healed in 3 months with no additional procedures. The patient went back to all activities of daily living in 6 months and was followed up for 2 years post-surgery. His preoperative AOFAS score improved from 58 to 76 . 

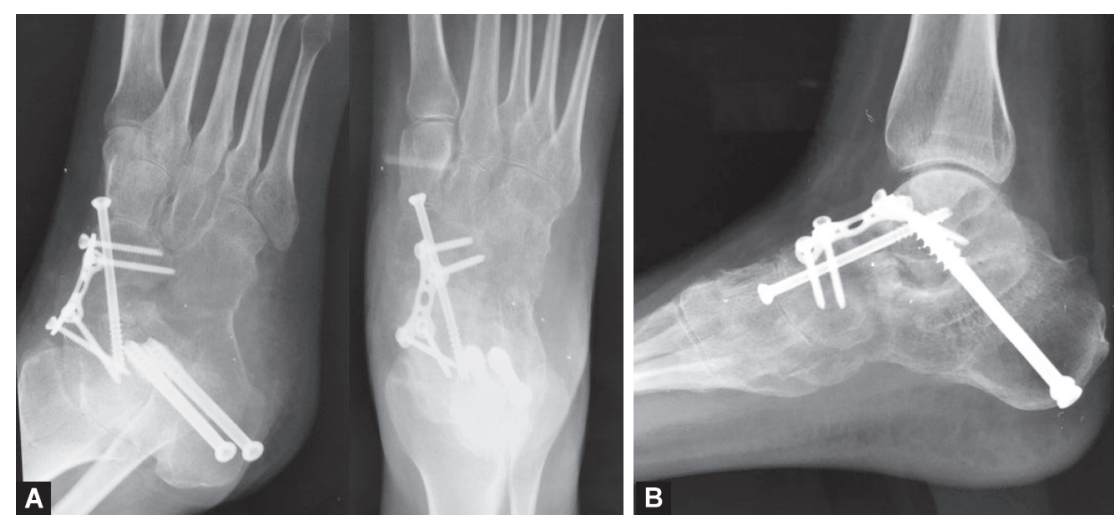

Figs $3 \mathrm{~A}$ and B: Case 1, follow-up at 18 months following corrective talonavicular fusion and medial calcaneal slide osteotomy

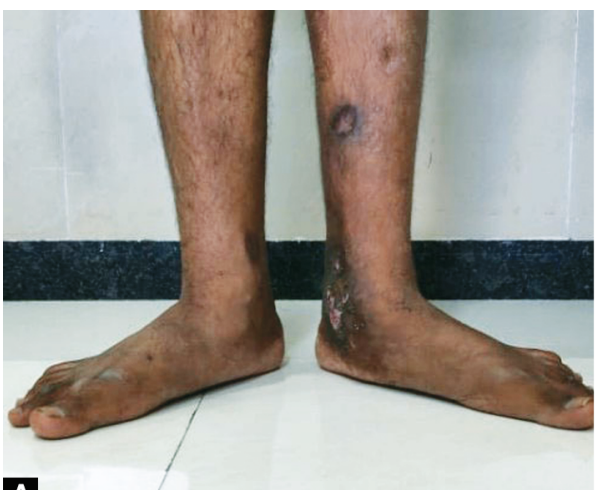

A

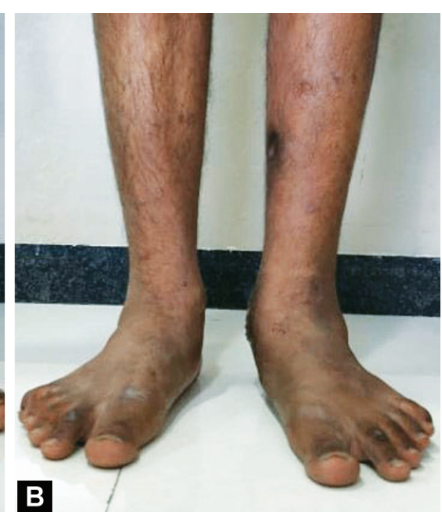

B

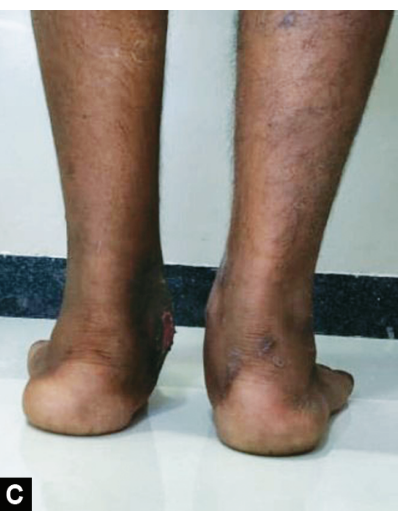

C

Figs 4A to C: Case 2, AG, an 18-year-old man with a non-healing ulcer over the medial aspect of left ankle, rigid flatfoot, and pes planovalgus deformity
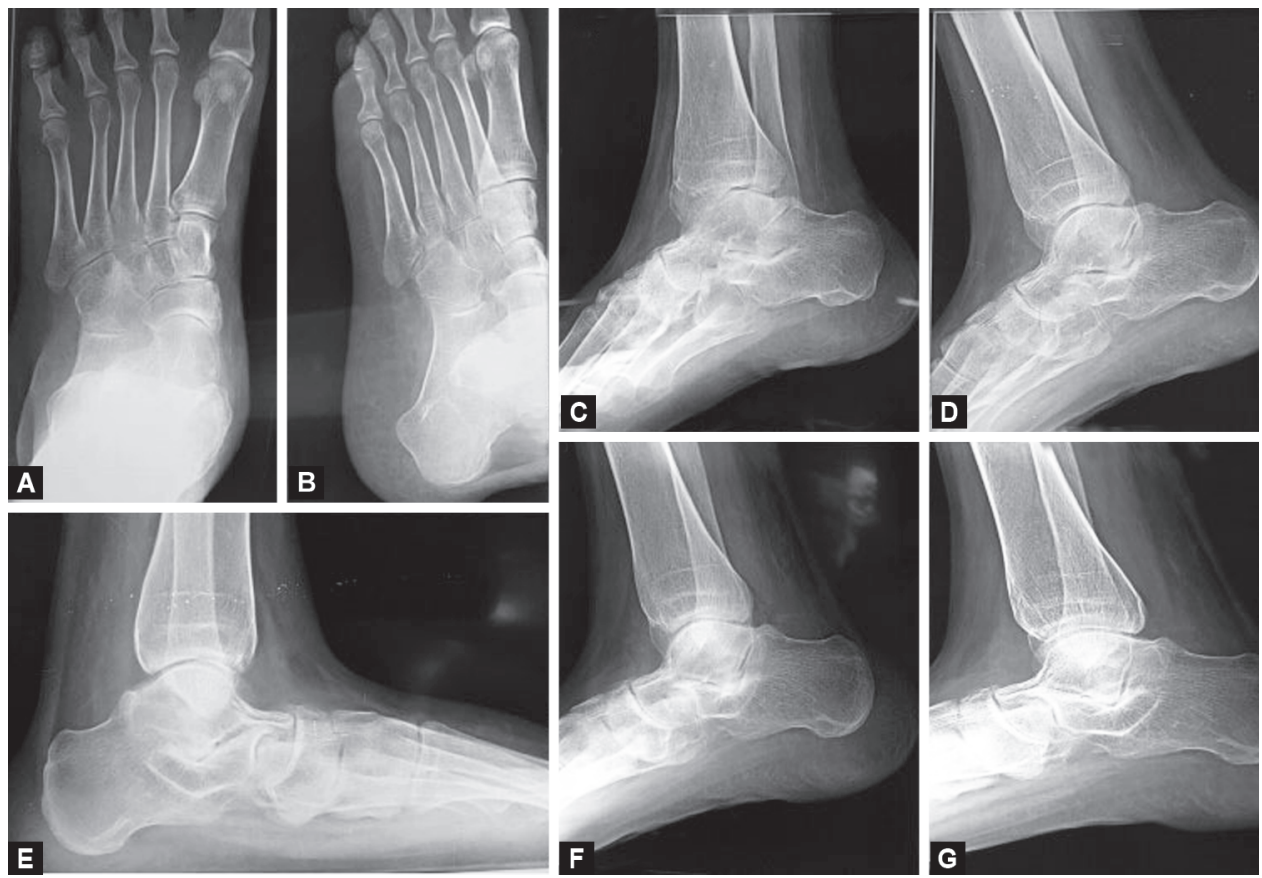

Figs 5 A to G: Case 2, preoperative radiographs demonstrating talocalcaneal and talonavicular coalitions

\section{Case 3}

AP, a 26-year-old doctor, presented with complaints of fatigue of his left foot while walking even with the medial arch support. He also complained of pain, usually by the end of the day. He was evaluated and noted to have a flat foot with heel in valgus. X-rays demonstrated talocalcaneal and calcaneonavicular coalitions and early arthritis around the talonavicular joint (Fig. 7). He underwent a medial calcaneal slide osteotomy, gastrocnemius recession, and talonavicular arthrodesis with a compression screw, and proximal tibia bone grafting (Fig. 8). At the end of 20 months follow-up, his 


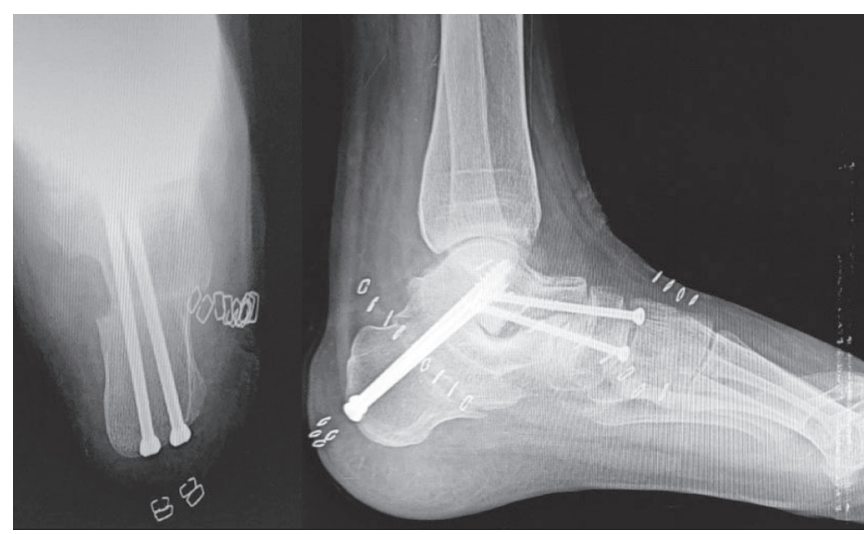

Fig. 6: Case 2, follow-up at 2 years following talonavicular fusion gastrocnemius recession with medial calcaneal slide osteotomy


Figs $8 \mathrm{~A}$ and B: Case 3 , follow-up at 20 months following talonavicular fusion with medial calcaneal slide osteotomy

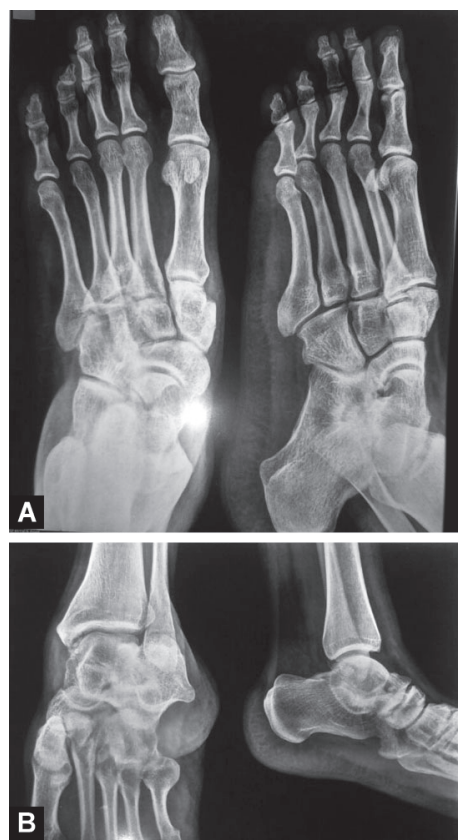

Figs 7A and B: Case 3, AP, a 26-year-old man, with rigid flatfoot and planovalgus deformity

walking pattern was similar to the opposite side without pain. His AOFAS score also improved to 82 from 64 preoperative.

\section{Case 4}

DG, a 40-year-old woman, presented to us with a complaint of severe pain over her left midfoot region, more while walking. Though her heel was in a neutral position, she was overloading the talonavicular joint with pes planus. Her X-rays showed a talocalcaneal coalition with a large impinging talonavicular osteophyte, contributing to anterior ankle impingement. As her subtalar joint was already fused, she underwent excision of the talonavicular osteophyte and cheilectomy of the talonavicular joint. She also underwent a gastrocnemius recession for gastrocnemius tightness. She was last seen at the end of 36 months, during which she was found to be walking comfortably without pain. Her AOFAS score improved to 78 in comparison to her preoperative score of 60.

\section{Case 5}

UP, a 32-year-old man, presented with severe left foot pain and fatigue while walking for 2 years. He was evaluated and found to have a talocalcaneal coalition with heel valgus, forefoot abduction, and gastrocnemius tightness. X-rays demonstrated arthritis changes around the talonavicular joint. He underwent gastrocnemius recession, Evans osteotomy, and medial calcaneal slide osteotomy followed by talonavicular fusion with a single cancellous screw and plate, augmented with cancellous bone graft. His foot biomechanics improved, and by 24 months, he was pain-free and walking comfortably with a postoperative AOFAS score of 76 .

\section{Discussion}

Incidence of tarsal coalitions ranges from 1 to $13 \%$, though actual incidence may be questionable as several cases are asymptomatic. Calcaneonavicular and talocalcaneal coalitions make up $90 \%$ of all tarsal coalitions. Calcaneonavicular coalition is generally present 
at 8-12 years, whereas patients with a talocalcaneal coalition present at around 12-16 years, with an equal sex predilection. ${ }^{4}$ The initial treatment is always conservative, intending to limit midfoot and hindfoot movements. Proven conservative modalities include nonsteroidal anti-inflammatory agents, physiotherapy, and orthotics. ${ }^{5}$ Operative treatment is indicated when conservative management fails. Currently, surgical techniques including bar resection, fat or extensor digitorum brevis interposition, and arthrodesis. ${ }^{6}$ In adults and those with arthritic changes, arthrodesis is preferred. ${ }^{7}$

Tarsal coalitions in adults usually present in a combination unlike isolated coalitions in pediatric and adolescent age groups. Also, they present in varied ways, most commonly with a variety of heel deformities, ${ }^{3}$ like we observed in four out of five patients, who had heel valgus. Though heel valgus is more common, tarsal coalitions also present with heel varus or neutral position. ${ }^{6}$ Anterior and posterior calcaneal osteotomies have been described for heel valgus correction in coalitions. ${ }^{6,8}$ If a pes planovalgus deformity in calcaneonavicular coalition, an Evans lateral lengthening calcaneal osteotomy can effectively correct the deformity and relieve stress. ${ }^{9}$

Adult patients with coalitions present with varying degrees of gastrosoleus tightness, early arthritic changes of the adjoining joints, secondary changes of flatfoot, and vasculitis or delayed healing of minor wounds on the contralateral surface of the deformity. Conservative treatment in such situations fails. This obligates treatment in adults to primarily focus on flat foot deformity correction and correction of the axis.

Gastrosoleus tightness causes compensatory stance and postural gait effects. The planovalgus deformity translates forces upward to the knee preventing full knee extension, exaggerates lumbar lordosis, and causes a shift in the center of balance during stance. Therefore, correction of the gastrocnemius contracture along with pes planovalgus deformity is necessary for realigning this postural malalignment. ${ }^{10}$

We also observed that one of our patients who presented with the talocalcaneal and calcaneonavicular coalition and planovalgus deformity had a non-healing ulcer on the medial aspect of the ankle, which failed to heal even after 2 years. Several case reports described delayed healing of wounds due to deformities, ${ }^{11}$ but a chronic non-healing ulcer due to rigid planovalgus deformity of the foot, to the best of our knowledge, has not been described in the literature. Mainly, the problem was the constant stretching of the skin while ambulation because of the faulty weight-bearing axis. In lieu of no literature support, a logical approach to correct the axis by correcting the flatfoot deformity was taken, which worked.

Several studies have shown that normal plantar pressures were not restored after isolated coalition resection in patients with accompanying flatfoot deformity in adult age. ' Studies also showed superior results with arthrodesis of joints vs excision of coalitions in the adult age group. ${ }^{1-7}$ Arthrodesis is a more predictable and final procedure compared with joint preserving surgeries. ${ }^{1-7}$ Earlier studies recommended treatment with triple or subtalar arthrodesis. Selective fusions for progressive planovalgus deformity secondary to the tarsal coalition are found to have favorable outcomes. ${ }^{6}$ In one of our cases, we did a joint preserving surgery in the form of cheilectomy, which seems to have worked in the short-term follow-up.

The main goals of arthrodesis are relief of pain from degenerated joints and restoration of the neutral axis of the foot and ankle. Triple (subtalar, talonavicular, calcaneocuboid) arthrodesis leads to stress transfer to the ankle joint, eventually causing degenerative arthrosis, an undesirable consequence. This forgoes calcaneocuboid fusions to spare uninvolved joints and lessen stress on the ankle joint. Hence, reserving triple arthrodesis for painful pan arthritis. ${ }^{12}$ More so, the calcaneal lengthening osteotomy is another desirable alternative to triple arthrodesis in hindfoot valgus with tarsal coalitions. ${ }^{8}$ In one of our cases, we corrected gross forefoot abduction with Evans osteotomy, salvaging the calcaneocuboid joint.

The presence of triple coalitions (talocalcaneal, calcaneocuboid, and talonavicular) is scarcely reported.$^{13}$ Conservative management is described in the literature for triple coalitions. One such case in our series was managed with corrective fusion at the talonavicular coalition.

This case series observed a varied presentation of tarsal coalitions in adults where some of the patients had more than one coalition and gastrocnemius contracture, even triple coalition, with either forefoot abduction, flatfoot deformity or non-healing ulcer; for which each patient had to be managed individually.

\section{Conclusion}

Symptomatic tarsal coalitions with planovalgus foot in adults need to be dealt with arthrodesis plus flat foot correction procedures. Limited arthrodesis has proved well that unaffected joints of the foot are spared to neutralize the stress transmitted to the ankle joint, thereby preventing arthritis of the ankle. Tarsal coalitions in adults can present with varieties of symptoms, and management approaches should be tailored.

\section{Clinical Significance}

Tarsal coalitions in adults can present in various forms, including heel valgus, forefoot abduction, and non-healing ulcers. The clinician needs to recognize such issues and treat them accordingly with arthrodesis and flatfoot deformity correction procedures.

\section{References}

1. Zhou B, Tang K, Hardy M. Talocalcaneal coalition combined with flatfoot in children: diagnosis and treatment: a review. J Orthop Surg Res 2014;9(1):129. DOI: 10.1186/s13018-014-0129-9.

2. Brennan SA, Kiernan C, Maleki F, et al. Talonavicular synostosis with lateral ankle instability-a case report and review of the literature. Foot Ankle Surg 2012;18(3):e34-e36. DOI: 10.1016/j.fas.2012.01.002.

3. Soni JF, Valenza W, Matsunaga C. Tarsal coalition. Curr Opin Pediatr 2020;32(1):93-99. DOI: 10.1097/MOP.0000000000000858.

4. Carr 2nd JB, Yang S, Lather LA. Pediatric pes planus: a state-of-the-art review. Pediatrics 2016;137(3):e20151230. DOI: 10.1542/peds.20151230.

5. Zaw H, Calder JD. Tarsal coalitions. Foot Ankle Clin 2010;15(2):349-364. DOI: 10.1016/j.fcl.2010.02.003.

6. Varner KE, Michelson JD. Tarsal coalition in adults. Foot Ankle Int 2000;21(8):669-672. DOI: 10.1177/107110070002100807.

7. Cohen BE, Davis WH, Anderson RB. Success of calcaneonavicular coalition resection in the adult population. Foot Ankle Int 1996;17(9):569-572. DOI: 10.1177/107110079601700912.

8. Mosca VS, Bevan WP. Talocalcaneal tarsal coalitions and the calcaneal lengthening osteotomy: the role of deformity correction. J Bone Joint Surg Am 2012;94(17):1584-1594. DOI: 10.2106/JBJS.K.00926.

9. Shang $L$, Wang $X$, Wang $A$, et al. Evans lateral lengthening calcaneal osteotomy in treatment of talocalcaneal coalition with hindfoot valgus deformity. Zhongguo Xiu Fu Chong Jian Wai Ke Za Zhi 2020;34(2):173-178.

10. Gougoulias N, O'Flaherty M, Sakellariou A. Taking out the tarsal coalition was easy: but now the foot is even flatter. What now? 
Foot Ankle Clin 2014;19(3):555-568. DOI: 10.1016/j.fcl.2014. 06.011.

11. Han G, Ceilley R. Chronic wound healing: a review of current management and treatments. Adv Ther 2017;34(3):599-610. DOI: 10.1007/s12325-017-0478-y.
12. Bohne WH. Tarsal coalition. Curr Opin Pediatr 2001;13(1):29-35. DOI: 10.1097/00008480-200102000-00005.

13. Farid A, Faber FWM. Bilateral triple talocalcaneal, calcaneonavicular, and talonavicular tarsal coalition: a case report. J Foot Ankle Surg 2019;58(2):374-376. DOI: 10.1053/j.jfas.2018.08.047. 